\title{
Escala Breve de Autocontrole (BSCS): Adaptação e Validação no Contexto Brasileiro
}

\author{
Gabriel Loureiro Figueiraํㅜㄹ Douglas de Farias Dutra, \\ Pedro Paulo Pires dos Santos, Bruno Figueiredo Damásio \\ Universidade Federal do Rio de Janeiro, Rio de Janeiro-RJ, Brasil
}

RESUMO

A Escala Breve de Autocontrole (BSCS) foi desenvolvida para avaliar diferentes níveis de autocontrole, uma capacidade individual de ativamente alterar os próprios desejos, comportamentos, emoções e pensamentos. Apesar da estabilidade do modelo unidimensional no estudo original, sua replicabilidade estrutural não foi corroborada pela literatura posterior. O presente estudo teve por objetivo oferecer informações sobre validade de construto em amostra brasileira, incluindo avaliação de modelos bidimensionais alternativos. A amostra foi composta por 405 indivíduos (61,2\% mulheres) com idades entre 18 e 46 anos $(M=22,32, D P=4,782)$. Foram realizadas análises fatoriais exploratórias (AFE) e confirmatórias (AFC), e a adequação do modelo foi avaliada por meio de índices de ajuste. Apenas um modelo emergiu satisfatoriamente. Os resultados são discutidos com base na literatura vigente e na relação da escala com outros instrumentos que fazem uso de diversas medidas do construto de autocontrole (Adiamento de Gratificação e Impulsividade).

Palavras-chave: autocontrole; autorregulação; impulsividade; análise fatorial; psicometria.

\section{ABSTRACT - Item Brief Self-Control Scale (BSCS): Psychometric Properties of the Brazilian Version}

The Brief Self-Control Scale (BSCS) was developed to measure the individual self-control spectrum, as a cognitive skill to actively alter one's own desires, behavior, emotions and thoughts. In the original validation, the BSCS displayed adequate psychometric properties for its unidimensional structure. However, this structure was difficult to replicate in subsequent studies. This study aimed to adapt and to assess evidence of validity of the measure for a Brazilian sample. The sample was composed of 405 individuals $(61.2 \%$ women) with ages ranging from 18 to 46 years $(M=22.32, S D=4.782)$. Exploratory (EFA) and confirmatory (CFA) factor analysis were conducted, and the adequacy of the model was assessed by means of model fit indexes. Only one model was found to be appropriate. The results are discussed based on recent scientific data and the relations between the scale and other instruments that evaluate diverse self-control measures (Delaying Gratification and Impulsivity).

Keywords: self-control; self-regulation; impulsivity; factor analysis; psychometrics.

\section{RESUMEN - Escala Breve de Autocontrol (BSCS): Adaptación y Validación en el Contexto Brasileño}

La Escala Breve de Autocontrol (BSCS) fue desarrollada para evaluar diferentes niveles de autocontrol, una capacidad individual alterar activamente la propia voluntad, comportamientos, emociones y pensamientos. En su estudio de validación, el instrumento presentó buenas propiedades psicométricas en su modelo unidimensional. No obstante, tal estructura no fue encontrada en investigaciones posteriores. El presente estudio tuvo como objetivo proporcionar información sobre la validez de constructo en una muestra brasileña, incluyendo la evaluación de modelos bidimensionales alternativos. Participaron de la muestra 405 individuos (61,2\% mujeres) con edades entre 18 y 46 años $(M=22,32, D S=4,782)$. Se realizaron análisis factoriales exploratorios $($ AFE) y confirmatorios (AFC). Se evaluó la adecuación del modelo por medio de índices de ajuste. Solo un modelo obtuvo resultados satisfactorios. Los resultados se discuten con base en la literatura vigente y en la relación de la escala con otros instrumentos que hacen uso de diversas medidas del constructo de autocontrol (Aplazamiento de la Gratificación e Impulsividad).

Palabras clave: autocontrol; autorregulación; impulsividad; análisis factorial; psicometría.

Nos seres humanos, o sistema volitivo é compreendido como instrumento fundamental para uma gama de funções psíquicas relacionadas à vontade e aos impulsos. Seu envolvimento inclui desde os mais simples como eliciamento e inibição de comportamentos até a tomada de decisão e concepção e execução de planos de ação. $\mathrm{O}$ sistema dessas estruturas do self denomina-se "autorregulação do indivíduo" (Baumeister, Bratslavsky, Muraven, \& Tice, 2018). A literatura segue em expansão na identificação de habilidades que compõem o construto da autorregulação, mas é teorizado que incluem um conjunto menor de traços e habilidades específicas, a exemplo 
o controle dos impulsos e o adiamento de gratificação (Baumeister, Heatherton, \& Tice, 1994; Baumeister, Vohs, \& Tice, 2007).

O autocontrole refere-se a uma capacidade individual de ativamente alterar os próprios desejos, comportamentos, emoções e pensamentos. Pode acontecer mediante necessidades ambientais (expectativas sociais, dinâmicas afetivas, situações problemáticas) ou demandas e esquemas internos do self (e.g., ideais, valores morais e éticos, planos, metas). Seu desenvolvimento evolutivo permitiu aos seres humanos expandirem o seu rol de comportamentos adaptativos (Gailliot et al., 2007; Gailliot \& Baumeister, 2018; Vohs \& Baumeister, 2018).

Autorregulação e seus correlatos são frequentemente estudados na literatura e tem um grande poder preditivo sobre a vida dos indivíduos: descrevem riscos de obesidade infantil (Seeyave et al., 2009), explicam aquisição e manutenção de comportamentos saudáveis visando longevidade (Joireman, Shaffer, Balliet, \& Strathman, 2012), são capazes de predizer boa adequação social (Tangney, Baumeister, \& Boone, 2004), assim como dependência de substâncias e incidência de comportamentos criminosos (Moffitt et al., 2011).

Reconhecidamente, um dos instrumentos psicométricos mais utilizados para mensuração do autocontrole é a Self-Control Scale (De Ridder, Lensvelt-Mulders, Finkenauer, Stok, \& Baumeister, 2012; Tangney et al., 2004). Sua versão breve de 13 itens (Brief Self-Control Scale) tem recebido atenção especial, devido ao seu aspecto econômico e aplicação. Apesar de sua conceitualização teórica como instrumento unidimensional (a), estudos posteriores que buscavam replicar com exatidão a estrutura fatorial original do instrumento, não obtiveram sucesso e, por isso, outras estruturas fatoriais foram hipotetizadas, a saber; (b) Ferrari, Stevens e Jason (2009); (c) Maloney, Grawitch e Barber, (2012); (d) De Ridder et al. (2012); e) Morean et al., (2014). Esses modelos também foram comparados entre si em amostras de outras localidades, porém, evidências ainda são insuficientes para rejeitar ou aceitar quaisquer modelos como única solução plausível para o construto (Lindner, Nagy, \& Retelsdorf, 2015).

O principal objetivo do atual estudo foi fornecer evidências de validade para uma versão da BSCS no contexto brasileiro, a luz do rigor técnico no processo de tradução e adaptação de instrumentos entre diferentes culturas (Borsa, Damásio, \& Bandeira, 2012). Adicionalmente, procurou-se elucidar as propriedades psicométricas do instrumento adaptado por meio de MEE (Modelagem por Equações Estruturais), comparando os diferentes modelos existentes (MacCallum \& Austin, 2000).

\section{Modelo Original Unidimensional (A)}

Tangney et al. (2004) descrevem a necessidade da criação de uma medida específica para avaliar o autocontrole, tendo em vista a incapacidade dos instrumentos disponíveis em avaliar adequadamente a habilidade de dominar ou modificar respostas internas do sujeito, irromper tendências comportamentais indesejadas (impulsividade). De forma geral, quebrar hábitos, resistir a tentações e manter a autodisciplina são manifestações comportamentais da capacidade do self de autorregular-se e o objetivo do instrumento é mensurar esses aspectos.

Durante o processo de construção da escala original, foram conduzidos dois estudos, ambos com estudantes de graduação. A essas amostras foram apresentados um composto de 93 itens com respostas em modelo Likert de cinco pontos que avaliava o autocontrole em cinco domínios propostos por Baumeister, Heatherton e Tice (1994); controle cognitivo, controle emocional, controle dos impulsos, performance e contenção de hábitos. Com base nos resultados obtidos foram conduzidos procedimentos para refinamento da escala: remoção de itens com baixa correlação com a escala toda, retirada de itens duplicados e que possuíam alta variação conforme o sexo do respondente.

O instrumento refinado com 36 itens (Self-Control Scale - SCS) foi avaliado posteriormente por meio de uma análise fatorial exploratória, porém, os procedimentos realizados descrevem uma análise por componentes principais com rotação varimax. Os métodos usados para determinar a quantidade de fatores a serem retidos foram o critério de Kaiser-Guttman (Kaiser, 1960) em que se retêm fatores cujo eigenvalue $>1$ e o teste de Cattell que avalia o ponto de inflexão ("cotovelo") no gráfico de scree plot (Cattell, 1966). Apesar dessa análise ter demonstrado uma estrutura de cinco fatores distintos para a BSCS, os diferentes fatores não foram capazes de melhorar a capacidade explicativa do instrumento. Por conta disso, os autores sugeriram a utilização do escore total para ambas as versões.

Com base na correlação entre itens dos estudos 1 e 2, também foi construída uma versão breve do instrumento (Brief Self-Control Scale), composta por 13 itens. As duas versões do instrumento apresentaram uma alta correlação entre si $(r=0,93)$ e bons índices de consistência interna ( $\alpha$ de Cronbach $=0,89$ e 0,85, nas versões longa e breve, respectivamente). Maiores dados sobre as propriedades psicométricas não foram reportados.

Tendo em vista o uso e importância da análise fatorial exploratória (AFE) para escalas psicométricas, é necessário estabelecer que a análise por componentes principais (ACP) difere-se da primeira, e que também os métodos utilizados na escala original (rotação ortogonal varimax, critério de Kaiser-Guttman e teste de scree plot) apresentam pouca acurácia no que tange à extração e retenção de fatores em variáveis latentes na psicologia (Damásio, 2012). Apesar disso, segundo os autores originais, os valores obtidos foram idênticos: tanto no uso de rotações ortogonais e oblíquas, quanto no uso de análise de componentes principais e eixo principal de fatoração (uma técnica de extração da AFE). 


\section{Método}

\section{Participantes}

A amostra foi composta por 405 brasileiros (61,2\% mulheres) com idades entre 18 e 46 anos $(M=22,32$, $D P=4,78)$. Deste total $54,1 \%$ eram solteiros, $37 \%$ estavam namorando e 5,9\% somavam os casados, noivos e morando juntos. Do total de participantes, 68,9\% disseram possuir alguma religião ou crença espiritual, todos os participantes estavam cursando o ensino superior sendo que entre estes 30,6\% disseram trabalhar concomitantemente. Além disto, 87,9\% da amostra afirmou que depende financeiramente de alguém. Trata-se de uma amostra por conveniência, de diferentes estados brasileiros, composta por 94,1\% de participantes do RJ, 3\% de estados variados (AM, BA, DF, RS, RO e SP) e 2,9\% que não declararam seu estado de origem. Para participar do estudo, era necessário ser brasileiro e maior de idade.

\section{Instrumentos}

Escala Breve de Autocontrole (BSCS). A BSCS (Brief Self-Control Scale, Tangney et al., 2004) é composta por 13 itens mensurados em uma escala do tipo Likert de cinco pontos, variando entre $1=$ "Não parece nada comigo" e 5 = "Parece totalmente comigo". Os escores da escala podem variar entre 5 e 65 . O processo de adaptação seguiu as diretrizes técnicas para adaptação transcultural e validação de instrumentos psicológicos (Borsa et al., 2012). A escala foi traduzida por dois tradutores independentes, sendo um brasileiro, fluente em língua inglesa, e um americano nativo, fluente na língua portuguesa. As duas traduções foram sintetizadas em uma única versão pela equipe de pesquisa, utilizando itens de ambas as traduções, que foram considerados mais adequados pelo time. Pequenos ajustes semânticos, idiomáticos e linguísticos foram realizados. Após a síntese das traduções e os referidos ajustes, a escala foi avaliada por um grupo de participantes, público-alvo $(n=12)$. Esses participantes responderam à uma ficha de avaliação da escala, que questionava sobre a compreensibilidade dos itens. Todos os itens foram considerados claros para todos os participantes, não havendo sugestão adicional de alterações.

Inventário de Adiamento de Gratificação (DGI-35). O DGI-35 (Delaying Gratification Inventory; Hoerger, Quirk, \& Weed, 2011; adaptada por Figueira, de Lemos, \& Damásio, no prelo) é um instrumento de autorrelato com 35 itens com respostas do tipo Likert variando entre $1=$ "Discordo totalmente" a $5=$ "Concordo totalmente". O adiamento de gratificações é uma habilidade componente da autorregulação, caracterizada pela escolha em adiar uma recompensa imediata por outra mais gratificante, em outro momento futuro. O instrumento avalia essa capacidade em cinco grandes facetas; comida (relacionado à alimentos instigantes), realização (relacionado à realização pessoal subjetiva), dinheiro (relacionado à recompensas financeiras), social (relacionado ao impacto de comportamentos nas relações humanas) e físico (relacionado a satisfações físicas). No presente estudo, foi utilizado o escore global da escala (Fidedignidade Composta $=0,953)$.

Escala de Impulsividade de Barratt (BIS-11). A BIS-11 (Barratt Impulsivity Scale, versão 11; Patton, Stanford, \& Barratt, 1995, adaptada por Malloy-Diniz et al., 2010) é uma escala de autorrelato composta por 30 itens, respondidos em uma escala tipo Likert de quatro pontos, a saber: 1 - "raramente ou nunca"; 2 = "de vez em quando"; 3 = "com frequência"; 4 = "quase sempre/sempre". Quanto maior o escore do respondente, maior o seu nível de impulsividade. A escala avalia as manifestações da impulsividade em três domínios: impulsividade motora, impulsividade atencional e impulsividade por não planejamento, com base no modelo teórico proposto por Ernst Barratt (1959). No presente estudo, foi utilizado o escore global da escala (Fidedignidade Composta =0,907).

Escala de Felicidade Subjetiva (SHS). A SHS (Subjective Happiness Scale - Lyubomirsky \& Lepper, 1999; adaptada por Damásio, Zanon, \& Koller, 2014) é um instrumento unidimensional composto por quatro itens em escala tipo Likert variando de 1 a 7, que avalia os níveis de felicidade subjetiva. Os escores dos sujeitos podem variar de quatro a 28 . No presente estudo, foi utilizado o escore global da escala (Fidedignidade Composta=0,859). Há evidências que a felicidade subjetiva funciona como facilitadora da autorregulação comportamental e emocional (Aspinwall, 1998; Keltner \& Bonanno, 1997).

Escala de Positividade (PS). A PS (Positivity Scale; Caprara et al., 2012; adaptada por Borsa, Damásio, Souza, Koller, \& Caprara, 2015) é um instrumento composto por oito itens em escala tipo Likert variando de 1 a 5 , que avaliam a visão positiva que as pessoas têm sobre si mesmas, sobre a vida e sobre o futuro. Os escores dos sujeitos podem variar de cinco a 40 . No presente estudo, foi utilizado o escore global da escala (Fidedignidade Composta $=0,856)$. A positividade está associada à maior flexibilidade e autorregulação cognitiva, auxiliando na implementação de estratégias de manejo frente estresse ou adversidade (Fredrickson \& Joiner, 2002).

Escala de Satisfação com a Vida (SWLS). A SWLS (Satisfaction With Life Scale; Diener, Emmons, Larsen, \& Griffin, 1985; adaptada por Gouveia, Milfont, Fonseca, \& Coelho, 2009) é uma escala unidimensional Likert composta por cinco itens, cujas respostas podem variar de $1=$ "Discordo totalmente" a $5=$ "Concordo totalmente". Os escores dos sujeitos podem variar entre 5 e 25 pontos, sendo que escores mais altos correspondem a uma maior satisfação com a vida. No presente estudo, foi utilizado o escore global da escala (Fidedignidade Composta $=0,863)$. O autocontrole é um preditor da satisfação com a vida (Hofmann, Luhmann, Fisher, Vohs \& Baumeister, 2014)

Marcadores Reduzidos de Personalidade (MRP). Os MRP (Hauck Filho, Machado, Teixeira, 
\& Bandeira, 2012) compõe um instrumento reduzido com 25 itens para mensurar os Cinco Grandes Fatores da Personalidade (CGF), derivado de um instrumento originalmente com 64 marcadores construído para a população brasileira (Hutz et al., 1998). Esse instrumento é composto por adjetivos divididos em cinco subescalas, que complementam o enunciado "Eu sou uma pessoa..." utilizando-se de uma escala Likert de cinco pontos, sendo $1=$ "Discordo totalmente" e $5=$ "Concordo totalmente". No presente estudo, foram utilizados os escore dos fatores de "Conscienciosidade" (Fidedignidade Composta $=0,813$ ) e "Neuroticismo" (Fidedignidade Composta $=0,781$ ).

\section{Procedimentos Éticos e de Coleta de Dados}

O estudo teve aprovação do Comitê de Ética do Centro de Filosofia e Ciências Humanas $(\mathrm{CFCH})$ da Universidade Federal do Rio de Janeiro. A coleta de dados aconteceu por meio de formulários on-line, nos quais participantes precisavam aceitar e concordar com o Termo de Consentimento Livre e Esclarecido (TCLE) para responder o questionário da pesquisa. A privacidade dos participantes, bem como o sigilo e confidencialidade dos dados foram garantidas. O link para o formulário de pesquisa foi divulgado por listas de e-mails e por redes sociais.

\section{Procedimentos de Análise dos Dados}

Primeiramente foi realizada uma análise fatorial exploratória (AFE) para investigar as características dimensionais da BSCS. O número de fatores a serem retidos foi determinado a partir das técnicas de análise paralela, com permutação aleatória dos dados (Timmerman \& Lorenzo-Seva, 2011), e Método Hull (Lorenzo-Seva, Timmerman, \& Kiers, 2011). Em seguida, a estrutura fatorial exploratória foi contrastada com outras estruturas sugeridas pela literatura, por meio de análises fatoriais confirmatórias (AFC). As análises fatoriais (AFE e AFC) foram implementadas com o método de estimação Weighted Least Squares Mean-and-Variance Adjusted (WLSMV, Muthén \& Muthén, 2017), recomendado para dados ordinais como escalas do tipo Likert. A adequação da estrutura fatorial aos dados observados foi avaliada por meio do teste do qui-quadrado e a razão entre qui-quadrado e graus de liberdade $\left(\chi^{2} / g l\right)$; Comparative Fit Index (CFI), Tucker-Lewis Index (TLI) e o Root-Mean-Square Error of Approximation (RMSEA). De acordo com os critérios utilizados (Brown, 2014), valores de CFI e TLI devem ser maiores que 0,90 , preferencialmente acima de 0,95 e valores de RMSEA (90\% IC) não devem ser superiores a 0,08 . Índices de fidedignidade da escala foram avaliados por meio da fidedignidade composta (FC), pois esta considera a variação das cargas fatoriais em oposição ao coeficiente alfa, onde há o pressuposto da tau-equivalência, que determina que as cargas são iguais entre si. Dessa forma, a FC apresenta-se como um indicador mais robusto de consistência interna (Valentini \& Damásio, 2016).

\section{Resultados}

A análise fatorial foi realizada sobre a matriz de correlações de policóricas entre os itens da BSCS. A matriz de correlação se mostrou adequada para o procedimento de análise fatorial, como indicado pelo índice $\mathrm{KMO}(0,85$ - considerado bom) e o teste de esfericidade de Bartlett $\left(\chi^{2}[78]=1131,5, p<0,05\right)$. Tanto a análise paralela quanto o método Hull indicaram a existência de um único fator latente. A retenção fatorial deve ocorrer antes do fator indicado em que a variância explicada dos dados aleatórios passe a ser maior do que a dos dados reais, sugerindo retenção espúria de fatores.

Tabela 1

Cargas Fatoriais da BSCS

\begin{tabular}{|c|c|}
\hline Itens & F1 \\
\hline BSCS1 & $-0,498$ \\
\hline BSCS2 & 0,417 \\
\hline BSCS3 & 0,507 \\
\hline BSCS4 & 0,405 \\
\hline BSCS5 & 0,620 \\
\hline BSCS6 & $-0,473$ \\
\hline BSCS7 & 0,507 \\
\hline BSCS8 & $-0,600$ \\
\hline BSCS9 & 0,671 \\
\hline BSCS10 & 0,552 \\
\hline BSCS11 & $-0,558$ \\
\hline BSCS12 & 0,653 \\
\hline BSCS13 & 0,542 \\
\hline Variância Explicada & 41,1 \\
\hline Fidedignidade Composta & 0,843 \\
\hline
\end{tabular}


Posteriormente, o modelo unifatorial (A, ver Tabela 1) resultante da AFE (proposto pelos autores originais da BSCS, Tangney et al., 2004) foi avaliado, e obteve um ajuste aceitável aos dados, conforme indicado pelos valores de $\chi^{2}$, CFI, TLI e RMSEA, e fidedignidade composta (FC) de 0,84 (Tabela 2). Entretanto, foram realizadas uma série de análises fatoriais confirmatórias (AFCs) com vistas a avaliar a plausibilidade de outros modelos propostos na literatura (De Ridder et al., 2012; Ferrari, Stevens, \& Jason, 2009; Maloney et al., 2012; Morean et al., 2014). O modelo bifatorial de 13 itens (B) proposto por Ferrari et al. (2009), que divide a escala nos fatores Autodisciplina (itens: 2, 3, 4, 5, 7, 9, 10, 12 e 13; FC=0,801) e Controle de Impulsos (itens: 1, $6,8,11 ; \mathrm{FC}=0,685)$ não obteve um ajuste adequado aos dados.

Tabela 2

Índices de Ajuste de Diferentes Modelos da BSCS

\begin{tabular}{ccccccc}
\hline & Modelo & $\chi^{2}(\mathrm{gl})$ & $\chi^{2} / g l$ & CFI & TLI & RMSEA (90\% IC) \\
\hline A & Um fator $^{\mathrm{a}}$ (13 itens) & $216,004(65)$ & 3,32 & 0,939 & 0,927 & $0,076(0,062-0,080)$ \\
B & Dois fatores $^{\mathrm{b}}$ (13 itens) & $283,690(64)$ & 4,43 & 0,894 & 0,871 & $0,092(0,081-0,103)$ \\
C & Dois fatores $^{\mathrm{c}}$ (8 itens) & $60,414(19)$ & 3,17 & 0,961 & 0,943 & $0,073(0,053-0,095)$ \\
D & Dois fatores $^{\text {(10 }}$ (1tens) & $197,977(34)$ & 5,82 & 0,882 & 0,844 & $0,109(0,095-0,124)$ \\
E & Dois fatores $^{\mathrm{e}}$ (7 itens) & $77,098(14)$ & 5,51 & 0,936 & 0,904 & $0,105(0,083-0,129)$ \\
F & Dois fatores $^{f}$ (6 itens) & $32,006(8)$ & 4,00 & 0,971 & 0,945 & $0,086(0,056-0,118)$ \\
\hline
\end{tabular}

Nota. A=Modelo unidimensional exploratório e proposto por Tangney et al. (2004). B=Modelo proposto por Ferrari et al. (2009). $\mathrm{C}=$ Modelo proposto por Maloney et al (2012). D=Modelo proposto por De Ridder et al. (2011). E=Modelo proposto por Morean et al. (2014). F: Modelo de Morean et al. (2014) sem o item 13

Dentre os modelos que eliminam itens da BSCS (C, D e E) apenas um dos modelos apresentou índices de ajuste aceitáveis. O modelo $(\mathrm{C})$ com fatores de Contenção (itens 1, 2, 7 e 8; FC=0,598) e Impulsividade (itens 5, 9, 12 e 13; FC=0,742) de Maloney, Grawitch e Barber (2012) foi aceitável em todos os critérios de ajuste aos dados. O modelo (D) com fatores de Inibição (itens $1,2,5,6,9$ e 12; FC=0,741) e Iniciação (itens 3, 10,11, e 13; FC $=0,634)$ de De Ridder et al. (2012) obteve todos os índices abaixo dos critérios de aceitação. O modelo (E) de Autodisciplina (itens 1,8 e 11, FC=0,64) e Controle dos Impulsos (itens 5, 9, 12 e 13; FC=0,74) proposto por Morean et al. (2014) obteve CFI e TLI aceitáveis, porém RMSEA elevado (Tabela 2).

Analisando-se os índices de modificação das soluções acima, notou-se uma correlação residual entre os itens
12 ("Às vezes não consigo deixar de fazer algo, mesmo sabendo que aquilo é errado") e 13 ("Frequentemente, ajo sem pensar em todas as alternativas") no modelo de Morean et al. (2014). Assim esse modelo foi testado novamente sem o item $13(\mathrm{~F})$, que possuía menor carga fatorial no modelo anterior (Item 12, $\lambda=0,709$; Item $13, \lambda=0,554)$. A eliminação do item 13 resultou em uma melhora em todos os índices de ajuste. Entretanto, houve redução na fidedignidade composta do fator de Impulsividade $(\mathrm{FC}=0,71)$ e os índices de RMSEA permaneceram fora do intervalo normalmente considerado como aceitável (estimativa abaixo de 0,08 com intervalo de confiança abaixo de 0,1; Brown, 2014; ver Tabela 2). Assim, conclui-se que o modelo unidimensional e o modelo de Maloney et al. (2012) são os mais ajustados para a amostra do presente estudo.

Tabela 3

Tabela de Correlação dos Instrumentos

\begin{tabular}{|c|c|c|c|c|c|c|c|c|}
\hline rho de Spearman & BSCS & DGI-35 & BIS-11 & SHS & PS & SWLS & MRP_Consc & MRP_Neuro \\
\hline BSCS & 1,000 & & & & & & & \\
\hline DGI-35 & $0,622^{* *}$ & 1,000 & & & & & & \\
\hline BIS-11 & $-0,636^{* *}$ & $-0,565^{* *}$ & 1,000 & & & & & \\
\hline SHS & $0,254^{* *}$ & $0,283^{* *}$ & $-0,108$ & 1,000 & & & & \\
\hline PS & $0,416^{* *}$ & $0,389^{* *}$ & $-0,210^{* *}$ & $0,574^{* *}$ & 1,000 & & & \\
\hline SWLS & $0,389^{* *}$ & $0,314^{* *}$ & $-0,225^{* *}$ & $0,501^{* *}$ & $0,628^{* *}$ & 1,000 & & \\
\hline MRP_Consc & $0,678^{* *}$ & $0,528^{* *}$ & $-0,520^{* *}$ & $0,295^{* *}$ & $0,381^{* *}$ & $0,335^{* *}$ & 1,000 & \\
\hline MRP_Neuro & $-0,346^{* *}$ & $-0,280^{* *}$ & $0,241^{* *}$ & $-0,441^{* *}$ & $-0,572^{* *}$ & $-0,336^{* *}$ & $-0,182^{* *}$ & 1,000 \\
\hline
\end{tabular}

Nota. ${ }^{* *}$ A correlação é significativa no nível 0,01 (duas extremidades) 
A BSCS correlacionou-se negativamente com a BIS-11 $(r=-0,636 ; p<0,01)$ e com Neuroticismo $(r=-$ $0,346 ; p<0,01)$. A BSCS correlacionou-se positivamente com as escalas DGI-35, SHS, PS, SWLS e com o fator de conscienciosidade dos MRP (Tabela 3). As correlações entre a BSCS, a BIS-11 e o DGI-35 estão de acordo com a literatura, visto que o autocontrole e o adiamento de gratificações são entendidos como habilidades componentes da autorregulação e a impulsividade é resultante da baixa capacidade autorregulatória (Nigg, 2017). O autocontrole também está relacionado com afetos positivos e satisfação com a vida, hipotetizando-se que um dos objetivos da utilização do autocontrole seja alcançar estados de bem-estar, por meio de afetos positivos (Aspinwall, 1998). As correlações entre a BSCS, a SHS, a PS e a SWLS reforçam a hipótese de que o autocontrole está relacionado com a felicidade e satisfação com a vida.

\section{Discussão}

Após as testagem e avaliação dos diferentes modelos existentes para a BSCS por intermédio de análises fatoriais exploratórias e confirmatórias, o modelo unidimensional original de Tangney et al. (2004) emergiu como ajustado aos dados da presente amostra. Apesar disso, outros modelos bifatoriais também foram testados, tendo em vista sua plausibilidade teórica e aparecimento consistente na literatura acerca do instrumento. Apenas os modelos de Maloney et al. (2012) e uma versão modificada do modelo de Morean et al. (2014) apresentaram índices aceitáveis.

Considerando o ajuste dos outros modelos bidimensionais, dois problemas concernem seu uso: (a) a remoção excessiva de itens do instrumento original pode ser prejudicial à validade de conteúdo e (b) ausência de evidências empíricas acerca dos fatores que compõem o autocontrole com base em um único instrumento. Com exceção do modelo de Ferrari et al. (2009), todos os outros modelos bidimensionais da BSCS propostos na literatura fazem remoção de alguns itens do instrumento. $\mathrm{Na}$ opinião dos autores, essa remoção pode apresentar-se como prejudicial à validade de conteúdo da escala caso o refinamento seja feito buscando atingir índices de ajuste mais adequados ou mediante a avaliação bruta de índices de modificação do modelo.

No exemplo aqui exposto, os modelos mais plausíveis entre os testados seriam o de Maloney et al. (2012) e o de Morean et al. (2014) com a remoção do item 13 (ver Tabela 2). Porém, como discutido, isso acarretaria um modelo com oito itens e outro modelo com apenas seis itens do total dos 13 originais. Outro ponto de importante ressalva é a ausência de unanimidade na composição teórica dos fatores do construto do autocontrole. No estudo original de Tagney, Baumeister e Boone (2004), os autores constroem os itens que compõem a
BSCS com base em uma literatura que sinaliza cinco domínios propostos por Baumeister et al. (1994); controle cognitivo, controle emocional, controle dos impulsos, performance e contenção de hábitos. Porém, apesar dessa construção com base teórica ter sido realizada, essa estrutura de cinco fatores não se apresentou como a mais parcimoniosa para a BSCS e para sua versão mais longa (SCS, 36 itens). Os próprios autores, inclusive, sugerem a utilização de ambas as escalas como instrumentos unidimensionais, tendo em vista essa problemática em suplantar as facetas.

Os modelos bidimensionais proposto por Maloney et al. (2012) e Morean et al. (2014) refletem a teoria de Carver (2005) em sua conceitualização dos domínios da contenção e impulsividade. O primeiro construto representaria a capacidade individual de deliberativamente ou disciplinadamente engajar-se em controle esforçado de si mesmo e o último estaria associado à espontaneidade de agir sob intuição e a tomadas de decisão heurísticas. Os modelos divergiram frente a escolha de itens diferentes na composição dos fatores.

Essa tentativa de adequar alternativas teóricas (Carver, 2005; Gray, 1994) a um instrumento cuja conceitualização e confecção dos itens foi realizada com base em outra teoria não apresenta-se como uma proposta adequada à construção e adaptação de instrumentos psicológicos, tendo em vista as definições constitutivas e operacionais dos construtos que fundamentaram a criação dos itens serem extensivamente diferentes, inclusive no número de fatores (Pasquali, 1998).

\section{Considerações Finais}

Tendo em vista a multiplicidade de teorias que explicariam os domínios do Autocontrole, a utilização do referido instrumento com características de construção teórica próprias corrobora a utilização em seu modelo unidimensional, como sugerido pelos autores originais. Para a utilização de outras teorias e fazendo jus a novas evidências empíricas sobre os domínios que compõe o Autocontrole, fazem-se necessários novos estudos com novos instrumentos de conceitualização própria, a fim de comparar as teorias e as facetas internas.

Para a amostra presente, foi possível aferir as qualidades psicométricas do instrumento assim como garantir a adequação do modelo adaptado unidimensional. $\mathrm{Na}$ opinião dos autores, essa é a melhor estrutura possível, pois não prejudica a mensuração do fenômeno reduzindo itens como os modelos alternativos propostos na literatura.

Mais estudos são recomendados, tendo em vista o interesse de compreender mais sobre o fenômeno na população brasileira e testar a estrutura do modelo teórico. Pesquisadores e demais interessados sobre o tema poderão se beneficiar da utilização deste instrumento (Anexo A) em suas práticas profissionais. 


\section{Referências}

Aspinwall, L. G. (1998). Rethinking the role of positive affect in self-regulation. Motivation and Emotion, 22(1), 1-32. doi: 10.1023/A:1023080224401 Barratt, E. S. (1959). Anxiety and Impulsiveness Related to Psychomotor Efficiency. Perceptual and Motor Skills, 9(3), 191-198. doi: 10.2466/ pms.1959.9.3.191

Baumeister, R. F., Bratslavsky, E., Muraven, M., \& Tice, D. M. (2018). Ego depletion: Is the active self a limited resource? Em R. Baumeister (Ed.), Self-Regulation and Self-Control (pp. 16-44). London, UK: Routledge. doi: 10.4324/9781315175775

Baumeister, R. F., Heatherton, T. F., \& Tice, D. M. (1994). Losing control: How and why people fail at self-regulation. San Diego, CA: Academic Press.

Baumeister, R. F., Vohs, K. D., \& Tice, D. M. (2007). The strength model of self-control. Current Directions in Psychological Science, 16(6), 351-355. doi:10.1111/j.1467-8721.2007.00534.x

Borsa, J. C., Damásio, B. F., \& Bandeira, D. R. (2012). Adaptação e validação de instrumentos psicológicos entre culturas: Algumas considerações. Paideia, 22(53), 423-432. doi:10.1590/1982-43272253201314

Borsa, J. C., Damásio, B. F., Souza, D. S., Koller, S. H., \& Caprara, G. V. (2015). Psychometric properties of the positivity scale - Brazilian version. Psicologia: Reflexão \& Crítica, 28(1), 61-67. doi: 10.1590/1678-7153.201528107

Brown, T. A. (2014). Confirmatory factor analysis for applied research. New York, NY: The Guilford Press.

Caprara, G. V., Alessandri, G., Eisenberg, N., Kupfer, A., Steca, P., Caprara, M. G.,... Abela, J. (2012). The positivity scale. Psychological Assessment, 24(3), 701-712. doi:10.1037/a0026681

Carver, C. S. (2005). Impulse and constraint: Perspectives from personality psychology, convergence with theory in other areas, and potential for integration. Personality and Social Psychology Review, 9(4), 312-333 doi: 10.1207/s15327957pspr0904 2

Cattell, R. B. (1966). The scree test for the number of factors. Multivariate Behavioral Research, 1(2), 245-276. doi:10.1207/s15327906mbr0102_10

Damásio, B. F. (2012). Uso da análise fatorial exploratória em psicologia. Avaliação psicológica, 11(2), 213-228. Recuperado de http://pepsic. bvsalud.org/pdf/avp/v11n2/v11n2a07.pdf

Damásio, B. F., Zanon, C., \& Koller, S. H. (2014). Validation and psychometric properties of the Brazilian version of the subjective happiness scale. Universitas Psychologica, 13(1), 17-24. doi: 10.11144/Javeriana.UPSY13-1.vppb

De Ridder, D., Lensvelt-Mulders, G., Finkenauer, C., Stok, M., \& Baumeister, R. F. (2012). Taking stock of self-control: A meta-analysis of how self-control affects a wide range of behaviors. Personality and Social Psychology Review, 16(1), 76-99. doi: 10.1177/1088868311418749

Diener, E. D., Emmons, R. A., Larsen, R. J., \& Griffin, S. (1985). The satisfaction with life scale. Journal of Personality Assessment, 49(1), 71-75. doi: 10.1207/s15327752jpa4901_13

Ferrari, J. R., Stevens, E. B., \& Jason, L. A. (2009). The role of self-regulation in abstinence maintenance: Effects of communal living on self-regulation. Journal of Groups in Addiction \& Recovery, 4(1-2), 32-41. doi: 10.1080/15560350802712371

Figueira, G. L., de Lemos, V. C., \& Damásio, B. F. (no prelo). Inventário de Adiamento de Gratificação (DGI-35): Propriedades Psicométricas da Versão Brasileira. Psico-USF.

Fredrickson, B. L., \& Joiner, T. (2002). Positive emotions trigger upward spirals toward emotional well-being. Psychological Science, 13(2), 172-175. doi: 10.1111/1467-9280.00431

Gailliot, M. T., \& Baumeister, R. F. (2018). The physiology of willpower: Linking blood glucose to self-control. Em R. F. Baumeister (Ed.), Self-Regulation and Self-Control (pp. 16-44). London, UK: Routledge. doi: 10.4324/9781315175775

Gailliot, M. T., Baumeister, R. F., DeWall, C. N., Maner, J. K., Plant, E. A., Tice, D. M., ... Schmeichel, B. J. (2007). Self-control relies on glucose as a limited energy source: Willpower is more than a metaphor. Journal of Personality and Social Psychology, 92(2), 325-336. doi:10.1037/0022-3514.92.2.325

Gouveia, V. V., Milfont, T., Fonseca, P. N., \& Coelho, J. A., P. M. (2009). Life satisfaction in Brazil: Testing the psychometric properties of the Satisfaction With Life Scale (SWLS) in Five Brazilian samples. Social Indicators Research, 90(2), 267-277. doi:10.1007/s11205-008-9257-0

Gray, J. A. (1994). Personality dimensions and emotion systems. Em P. Ekman \& R.J. Davidson (Eds.), The nature of emotion (pp. 329-331). New York, NY: Oxford University Press.

Hauck Filho, N., Machado, W. D. L., Teixeira, M. A. P., \& Bandeira, D. R. (2012). Evidências de validade de marcadores reduzidos para a avaliação da personalidade no modelo dos Cinco Grandes Fatores. Psicologia: Teoria e Pesquisa, 28(4), 417-423. doi: 10.1590/S010237722012000400007

Hoerger, M., Quirk, S. W., \& Weed, N. C. (2011). Development and validation of the Delaying Gratification Inventory. Psychological Assessment, 23(3), 725-738. doi:10.1037/a0023286

Hofmann, W., Luhmann, M., Fisher, R. R., Vohs, K. D., \& Baumeister, R. F. (2014). Yes, but are they happy? Effects of trait self-control on affective well-being and life satisfaction. Journal of Personality, 82(4), 265-277. doi: 10.1111/jopy.12050

Hutz, C. S., Nunes, C. H. S. S., Silveira, A. D., Serra, J., Anton, M., \& Wieczorek, L. S. (1998). O desenvolvimento de marcadores para a avaliação da personalidade no modelo dos cinco grandes fatores. Psicologia: Reflexão e Crítica, 11(2), 395-411. doi: 10.1590/S010279721998000200015

Joireman, J., Shaffer, M. J., Balliet, D., \& Strathman, A. (2012). Promotion orientation explains why future-oriented people exercise and eat healthy: Evidence from the two-factor consideration of future consequences-14 scale. Personality and Social Psychology Bulletin, 38(10), 1272-1287. doi.org/10.1177/0146167212449362

Kaiser, H. F. (1960). The application of electronic computers to factor analysis. Educational and Psychological Measurement, 20(1), 141-151. doi: $10.1177 / 001316446002000116$

Keltner, D., \& Bonanno, G. A. (1997). A study of laughter and dissociation: Distinct correlates of laughter and smiling during bereavement. Journal of Personality and Social Psychology, 73(4), 687-702. doi: 10.1037//0022-3514.73.4.687

Lindner, C., Nagy, G., \& Retelsdorf, J. (2015). The dimensionality of the Brief Self-Control Scale- An evaluation of unidimensional and multidimensional applications. Personality and Individual Differences, 86(2015), 465-473. doi: 10.1016/j.paid.2015.07.006

Lorenzo-Seva, U., Timmerman, M. E., \& Kiers, H. A. L. (2011). The Hull method for selecting the number of common factors. Multivariate Behavioral Research, 46(2), 340-364. doi: 10.1080/00273171.2011.564527

Lyubomirsky, S., \& Lepper, H. S. (1999). A measure of subjective happiness: Preliminary reliability and construct validation. Social Indicators Research, 46(2), 137-155. doi:10.1023/A:1006824100041

MacCallum, R. C., \& Austin, J. T. (2000). Applications of Structural Equation Modeling in Psychological Research. Annual Review of Psychology, 51(1), 201-226. doi: 10.1146/annurev.psych.51.1.201

Malloy-Diniz, L. F., Mattos, P., Leite, W. B., Abreu, N., Coutinho, G., Paula, J. J. D., ... \& Fuentes, D. (2010). Tradução e adaptação cultural da Barratt Impulsiveness Scale (BIS-11) para aplicação em adultos brasileiros. Jornal Brasileiro de Psiquiatria, 59(2), 99-105. doi: 10.1590/ S0047-20852010000200004 
Maloney, P. W., Grawitch, M. J., \& Barber, L. K. (2012). The multi-factor structure of the Brief Self-Control Scale: Discriminant validity of restraint and impulsivity. Journal of Research in Personality, 46(1), 111-115. doi: 10.1016/j.jrp.2011.10.001

Moffitt, T. E., Arseneault, L., Belsky, D., Dickson, N., Hancox, R. J., Harrington, H., ... Caspi, A. (2011). A gradient of childhood selfcontrol predicts health, wealth, and public safety. Proceedings of the National Academy of Sciences, 108(7), 2693-2698. doi: 10.1073/ pnas.1010076108

Morean, M. E., DeMartini, K. S., Leeman, R. F., Pearlson, G. D., Anticevic, A., Krishnan-Sarin, S., ... O’Malley, S. S. (2014). Psychometrically improved, abbreviated versions of three classic measures of impulsivity and self-control. Psychological Assessment, 26(3), 1003-1020. doi: 10.1037/pas0000003

Muthén, L. K., \& Muthén, B. O. (2017). MPlus User's Guide. Eighth Edition. Los Angeles, CA: Múthen \& Múthen.

Nigg, J. T. (2017). Annual Research Review: On the relations among self-regulation, self-control, executive functioning, effortful control, cognitive control, impulsivity, risk-taking, and inhibition for developmental psychopathology. Journal of Child Psychology and Psychiatry, 58(4), 361-383. doi: 10.1111/jcpp.12675

Pasquali, L. (1998). Princípios de elaboração de escalas psicológicas. Revista de Psiquiatria Clínica, 25(5), 206-213.

Patton, J. H., Stanford, M. S., \& Barratt, E. S. (1995). Factor structure of the barratt impulsiveness scale. Journal of Clinical Psychology, 51(6), 768-774. doi: 10.1002/1097-4679(199511)51:6<768::AID-JCLP2270510607>3.0.CO;2-1

Seeyave, D. M., Coleman, S., Appugliese, D., Corwyn, R. F., Bradley, R. H., Davidson, N. S., ... Lumeng, J. C. (2009). Ability to Delay Gratification at Age 4 Years and Risk of Overweight at Age 11 Years. Archives of Pediatrics \& Adolescent Medicine, 163(4), $303-308$. doi:10.1001/archpediatrics.2009.12

Tangney, J. P., Baumeister, R. F., \& Boone, A. L. (2004). High self-control predicts good adjustment, less pathology, better grades, and interpersonal success. Journal of Personality, 72(2), 271-324. doi: 10.1111/j.0022-3506.2004.00263.x

Timmerman, M. E., \& Lorenzo-Seva, U. (2011). Dimensionality assessment of ordered polytomous items with parallel analysis. Psychological Methods, 16(2), 209-220. doi: 10.1037/a0023353

Valentini, F., \& Damásio, B. F. (2016). Variância Média Extraída e Confiabilidade Composta: Indicadores de Precisão. Psicologia: Teoria e Pesquisa, 32(2), 1-7. doi: 10.1590/0102-3772e322225

Vohs, K. D., \& Baumeister, R. F. (2018). Strength model of self- regulation as limited resource: assessment, controversies, update. Em R. F. Baumeister (Ed.), Self-Regulation and Self-Control (pp. 78-128). London, UK: Routledge. doi: 10.4324/9781315175775-10

Anexo A

Lista de itens da BSCS

\begin{tabular}{cr}
\hline Itens & Brief Self-Control Scale \\
\hline BSCS01 & Tenho facilidade em resistir a tentações. \\
BSCS02* & Tenho dificuldade em acabar com maus hábitos. \\
BSCS03* & Sou preguiçoso. \\
BSCS04* & Costumo dizer coisas inapropriadas. \\
BSCS05* & Faço certas coisas que podem me prejudicar, se elas forem divertidas. \\
BSCS06 & Recuso coisas que são prejudiciais a mim. \\
BSCS07* & Gostaria de ser mais disciplinado. \\
BSCS08 & As pessoas costumam dizer que sou muito disciplinado. \\
BSCS09* & Prazer e diversão às vezes me impedem de completar minhas tarefas. \\
BSCS10* & Tenho dificuldade para me concentrar. \\
BSCS11 & Consigo trabalhar de forma eficaz em direção a objetivos de longo-prazo. \\
BSCS12* & Às vezes não consigo deixar de fazer algo, mesmo sabendo que aquilo é errado. \\
BSCS13* & Frequentemente, ajo sem pensar em todas as alternativas. \\
\hline
\end{tabular}

Nota. Os itens marcados com * são inversamente codificados. As opções de resposta são: 1=Não parece nada comigo; 2=Não parece comigo; 3=Parece mais ou menos comigo; 4=Parece comigo, 5=Parece totalmente comigo

recebido em agosto de 2019 aprovado em outubro de 2019

\section{Sobre os autores}

Gabriel L. Figueira é psicólogo e mestrando em Psicologia (UFRJ). Atualmente é professor do Centro Universitário Celso Lisboa e da UNISUAM. Sócio-fundador da empresa Humanidados e membro do Laboratório de Psicometria e Psicologia Positiva (LP3)

Douglas Dutra é psicólogo (UFRJ) e mestre em Psicologia (UFRJ). Atualmente é consultor em análise de dados na Humanidados.

Pedro Paulo Pires é psicólogo (UFRJ, 2010), mestre (UFRJ, 2013) e doutor em Psicologia (UFRJ, 2015), É professor do Programa de Pós-Graduação em Psicologia da UFRJ e Chefe do Departamento de Psicometria do Instituto de Psicologia da UFRJ e Membro do corpo de docentes de Associação de Terapias Cognitivas do Rio de Janeiro.

Bruno Damásio é psicólogo (UEPB), mestre e doutor em Psicologia (UFRGS). Atualmente é professor do Programa de Pós-Graduação em Psicologia da UFRJ e coordenador do Laboratório de Psicometria e Psicologia Positiva (LP3). 